\title{
COLLARÍN PARA SOPORTES METÁLICOS
}

\author{
(COLLAR FOR METAL SUPPORTS)
}

José Luis de Miguel, Dr. Arquitecto

Escuela Técnica Superior de Arquitectura de Madrid. Dpto. de Estructuras

ESPAÑA

Fecha de recepción: 17-VI-98

\begin{abstract}
RESUMEN
En las obras de arquitectura los cálculos estructurales suelen ser pocos y sencillos, más bien para corroborar algo que se sabía de antemano. Los números se hacen, en su mayor parte, para explorar soluciones; cuando se trabaja en un caso particular debe operarse en un marco conocido, con valores predictables y soluciones tabuladas, sin sorpresas. Hacer números para fiarse a pies juntillas de ellos, es como una cita a ciegas, algo no recomendable en estructuras. Este articulo muestra cómo usar los conocimientos estructurales para explorar una solución: la de un collarín para soportes metálicos.
\end{abstract}

\section{SUMMARY}

In arichitectural works, structural calculuses are usually few and simple, they mostly corroborate what is already known. Calculations are made for the most part with the purpose of exploring solutions. When working in a specific case operations ought to be framed in the practical understanding, with predictable values and tabulated solutions, with no surprises. To write numbers and trust them blindly, is like a reckless appointment, something that should not be done in structures. This article shows how to use the structural knowledge in order to explore a specific solution : a collar for metal supports.
Antes de calcular propiamente un edificio hay que decidir material de los soportes y vigas, situación de los primeros y dirección de las segundas, sección de cada elemento y canto y tipo del forjado; más vale saber de antemano qué repercusión tendrá cada una de esas decisiones en los cálculos posteriores. Los números deben hacerse sólo, y con muchas precauciones, en los pocos temas en que no haya experiencia previa; si no sabes lo que va a salir, más vale no calcular. Este artículo muestra cómo usar los conocimientos estructurales para explorar una solución: la de un collarín para soportes metálicos.

Cada vez es más usual que los soportes de edificios sean de acero. En las obras urbanas abundan hoy en día casas de muy pocas plantas, tres o cuatro a lo sumo, en las que es poco probable llegar a rentabilizar un soporte de hormigón. Con el material típico de estos años, $\mathrm{H}-175$, la resistencia de cálculo es $175 / 1,5=116 \mathrm{kp} / \mathrm{cm}^{2}$ y la segura $116 / 1,6=73 \mathrm{kp} / \mathrm{cm}^{2}$. La tensión media no

(c) Consejo Superior de Investigaciones Científicas Licencia Creative Commons 3.0 España (by-nc) supera $73 \mathrm{x} 0,85=62 \mathrm{kp} / \mathrm{cm}^{2}$, que -en soportes- se reduce a $62 / 1,1=56 \mathrm{kp} / \mathrm{cm}^{2}$ y considerando la excentricidad mínima, en la sección completa no se puede pasar de $56 / 1,12=50 \mathrm{kp} / \mathrm{cm}^{2}$. El soporte mínimo, de tamaño A4, (20x30), es capaz de soportar, con toda seguridad, $20 \times 30 \times 50=30.000 \mathrm{kp}=30 \mathrm{t}$. Eso es mucho.

Una planta típica de viviendas, de $10 \times 10 \mathrm{~m}$, considerando $0,7 \mathrm{t} / \mathrm{m}^{2}$ de carga total y $0,7 \mathrm{kp} / \mathrm{m}$ de cerramiento en el contorno, pesa del orden de $10^{2} \times 0,7+40 \times 0,7=98 \mathrm{t}$. Si se disponen soportes cada $5 \times 5 \mathrm{~m}$, entre los nueve, aunque sean de sección mínima, son capaces de soportar 30x9=270 t. Aunque los soportes interiores se rentabilizan antes, hasta tres plantas no es, pues, plenamente eficaz la solución completa de soportes de hormigón. Por debajo de tres plantas un diseñador responsable acudirá a soportes metálicos. Con $\mathrm{H}-200$ la conclusión es aún más nítida y si el mínimo sube a H-250, habrá que superar cuatro plantas para que se justifique el soporte de hormigón. Eso significa 
que durante dos o tres plantas más las diferencias serán pequeñas y la menor superficie ocupada en planta y la rapidez de ejecución concederán ventaja, todavía, al soporte de acero. Paradójicamente, el aumento de resistencia del hormigón tenderá a hacer desaparecer la solución de soportes de hormigón en las obras de arquitectura de altura habitual. Con soportes de acero es más fácil ajustarse a las pequeñas compresiones de los edificios que más abundan.

Un perfil $\mathrm{H}$ tiene una fuerte asimetría, resultando muy penalizado por pandeo, salvo en los casos en los que la longitud de pandeo es diferente en los dos planos. Con 3,00 m de longitud de pandeo, el mínimo, tal como el HEB100, soporta con seguridad del orden de $18 \mathrm{t}$, pero invirtiendo $26 \mathrm{~cm}^{2}$ de sección. Su eficacia es muy baja. Un tubo $140 \times 140 \times 5$, con la misma sección y longitud que un HEB 100, soporta con seguridad $33 \mathrm{t}$. Y para ajustarse a compresiones menores, hay tubos desde $40 \mathrm{~mm}$ de lado, aunque, por razones prácticas, en obras de arquitectura rara vez se baja del de $80 \mathrm{~mm}$. Existiendo además tubos con diversas combinaciones de lado y espesor, se puede decidir lo uno por unas consideraciones, ajustando, posteriormente, lo segundo, por otras. Un perfil $\mathrm{H}$ es rentable cuando lo es, más aún, un soporte de hormigón. El soporte metálico por excelencia es, pues, el tubo.

Con soportes metálicos, los problemas más peliagudos provienen del engarce con el forjado, que, para las luces y uso citados, sigue siendo más eficaz en hormigón armado. Disponer una chapa en la base del fuste, anclada a la cara superior de un forjado, rematado en otra anclada en la cara inferior del siguiente (Figura 1) aunque simplifica la sustentación del forjado, que se limita a descansar sobre la placa inferior, es una solución muy compleja de replanteo y puesta en obra. La sencillez del montaje pasa por disponer cada fuste desde la cara superior de un forjado a la del siguiente. Pero eso obliga a la carga del forjado a acometer de lado, necesitando un artilugio para lograrlo. Disponer un corte y una chapa bajo el forjado encarece la solución, y no aumenta mucho la capacidad de recibir carga (Figura 2). A razón de unos $70 \mathrm{kp} / \mathrm{cm}^{2}$ de carga transversal, una chapa de acero alcanza el límite de su capacidad a flexión cuando la luz de vuelo supera tres veces su grueso. Eso da muy poco margen. La solución clásica suele ser la de un accesorio soldado al fuste pasante, que permite recoger la carga. El más reproducido en la literatura suele ser el de dos o cuatro perfiles UPN en cruceta (Figura 3), muy difíciles de embrochalar, y que necesita variantes para el caso de soportes próximos al borde del forjado.

En la Escuela Técnica Superior de Arquitectura de Madrid se viene explicando una solución usada y ensayada ampliamente desde hace un par de docenas de años'. En algunos ámbitos se la conoce con el nombre de "collarín ETSAM". Se trata de un perfil de ángulo, PNL, dispuesto al bies en derredor del fuste. La carga entra por las caras superiores

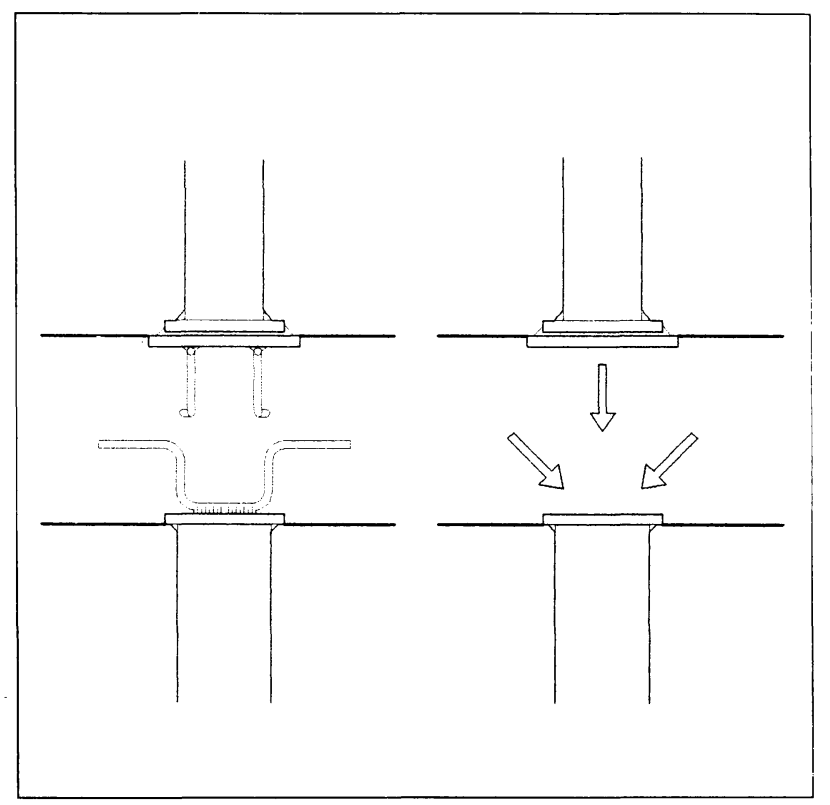

Figura 1.- Solución de fustes separados.

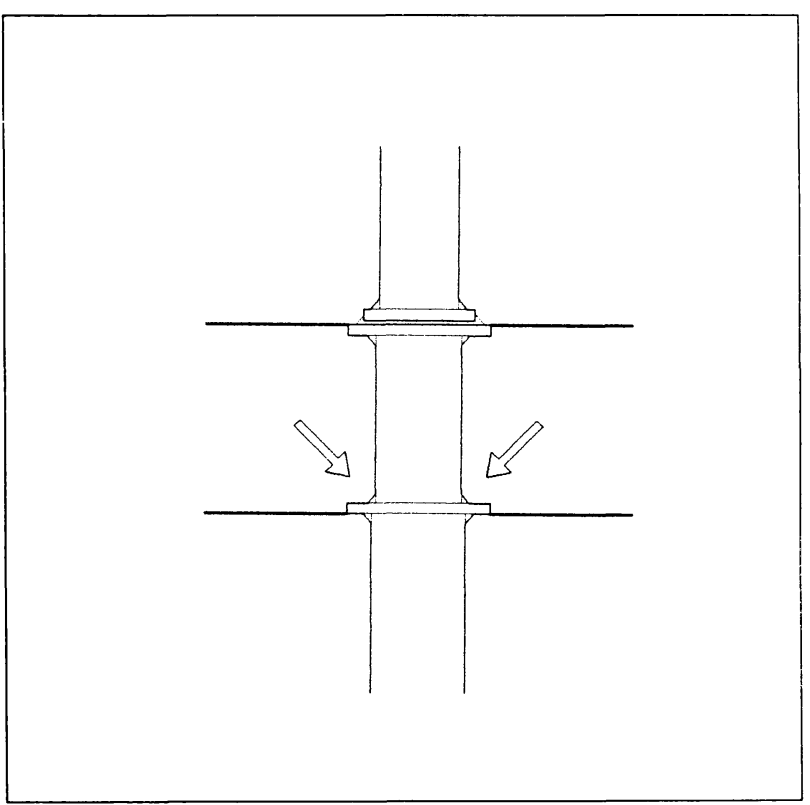

Figura 2.- Solución de placa intermedia.

del ángulo y el resalto que éste produce, impide el deslizamiento del forjado. Como sucede en muchos otros problemas, las indicaciones de $\mathrm{EH}$ no suministran la formulación particular aplicable al caso, sino sólo criterios generales que deben interpretarse. Tratar este problema en términos de cortante es torpe, ya que incita a ver la entrada de carga como una acción vertical. Como es bien sabido, en las proximidades de un apoyo interior, la solicitación de momento se traduce en tracción superior y compresión inferior; ésta, con el cortante, da una resultante oblicua (Figura 4), que es la biela por la que acomete propiamente la carga al soporte. La posición del ángulo del collarín ETSAM es sumamente favorable para eso. 


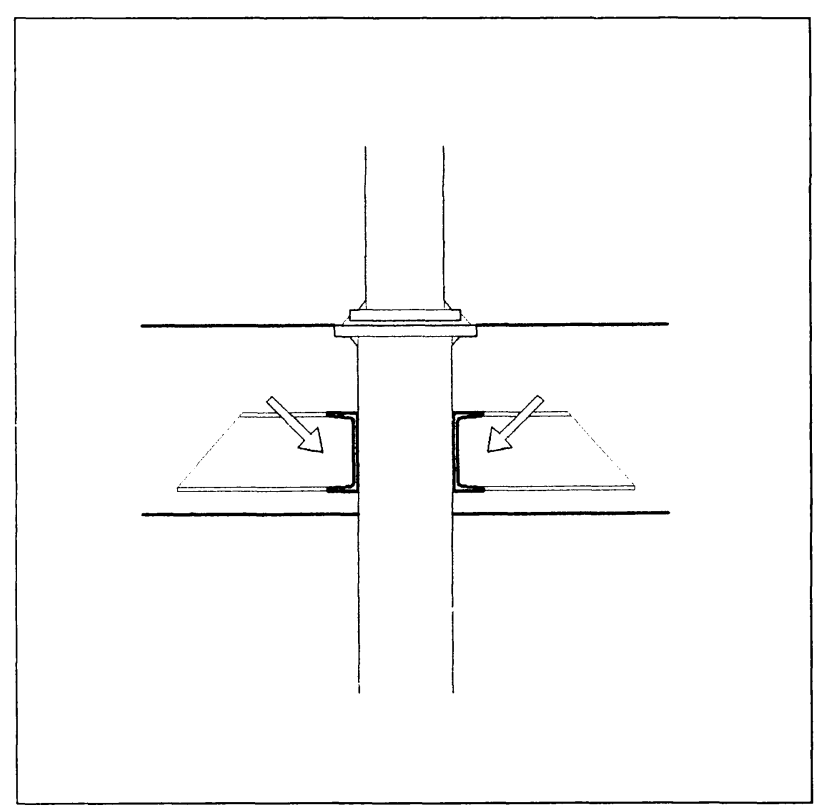

Figura 3.- Solución con perfiles UPN.

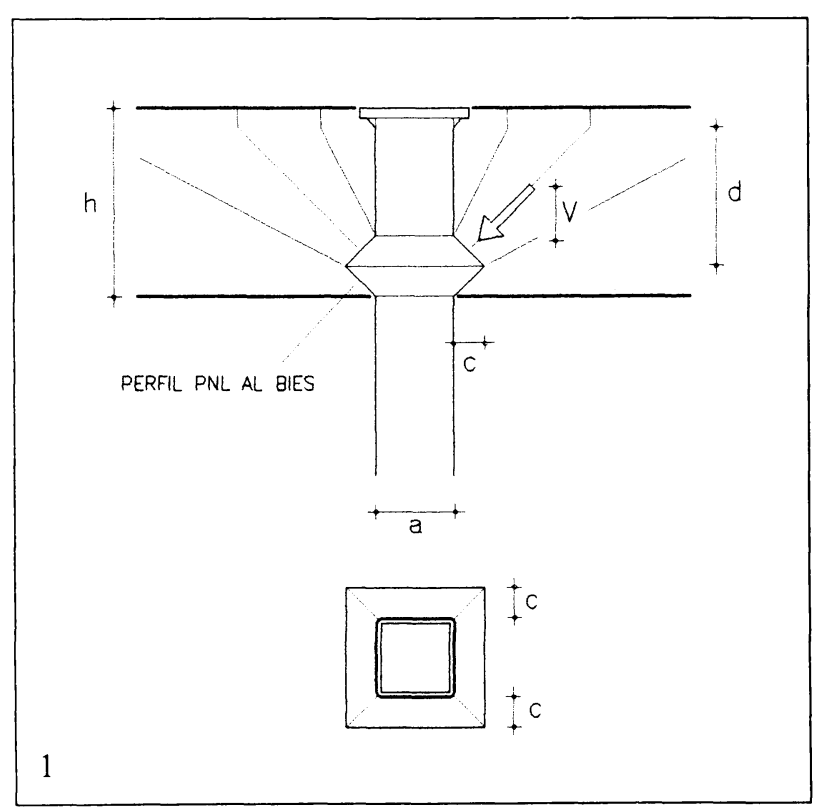

Figura 5.- 1) Collarin. 2) Comprobaciones de cortante y aplastamiento.

La capacidad del forjado en torno al soporte se tasa por la resistencia al punzonado. Aunque en rigor se trata de una comprobación a tracción de una sección troncopiramidal, se suele formular en su equivalente a tensión tangencial en una superficie cilíndrica cuya traza se conoce como perimetro crítico, se sitúa a medio canto ${ }^{2}$ mecánico del borde del área cargada. En este caso (Figura 5) dado que las bielas deben descargar en el collarín, el área cargada es, evidentemente, un cuadrado ${ }^{3}$ de lado $a+2 \cdot c$ y el canto, a efectos de la superficie resistente, debe deducirse de un grueso h-c. En ausencia de estribos computables a punzonado, la tensión media de cálculo en esa superficie crítica debe ser inferior a $2 \mathbf{f}_{\mathrm{ci}}$.

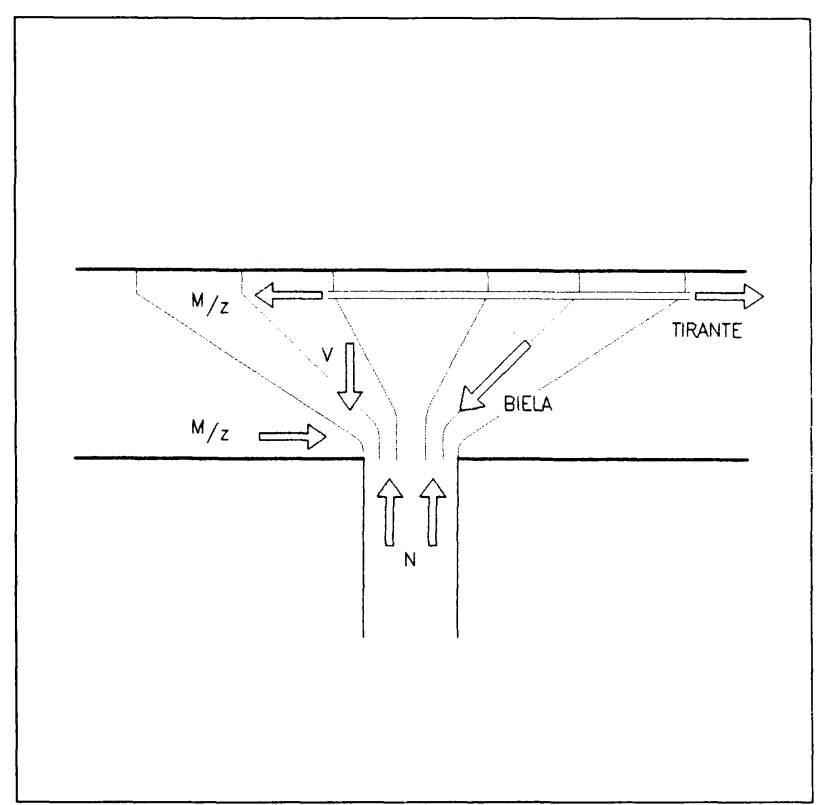

Figura 4.- Bielas en el nudo

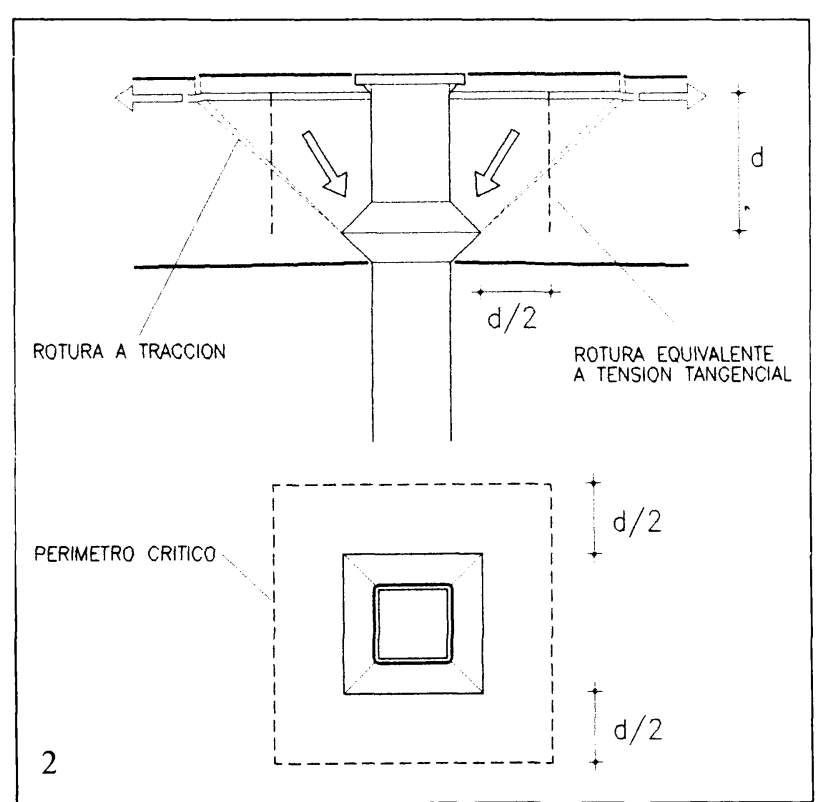

Sea un soporte que recoge $5 \times 5 \mathrm{~m}$ de planta de vivienda. Para la luz de 5,0 m, el canto óptimo sería 5,0/29=0,18 m. Para ese canto el peso de la planta puede ser de sólo $0,65 \mathrm{t} / \mathrm{m}^{2} \mathrm{y}$, por tanto, la carga que entra en el soporte es de $0,65 \times 25=16,2 \mathrm{t}$. Para fuste se prevé un tubo de $80 \mathrm{~mm}$ de lado y un collarín de PNL 40. El grueso neto es $18-2,8=15,2 \mathrm{~cm}$. En esta zona, con vigas planas, hay hasta cinco recubrimientos diferentes, uno para cada familia de armado (dos de malla, negativos del forjado, estribos de viga y armadura longitudinal de viga). El que opera en este caso es el del comportamiento de losa, es decir, el intermedio entre el de la armadura del forjado y de la viga, que puede estimarse en unos $3 \mathrm{~cm}$. El perímetro crítico se 
trazará, pues, a $(15,2-3,0) / 2=6,1 \mathrm{~cm}$, resultando un cuadrado de $8+(2,8 \times 2)+(6,1 \times 2)=25,8 \mathrm{~cm}$ de lado. La sección crítica tiene entonces $25,8 \times 4 \times 12,2=1.259 \mathrm{~cm}^{2}$.

La resistencia de cálculo de un hormigón $\mathrm{H}-175$ es $175 / 1,5=116 \mathrm{kp} / \mathrm{cm}^{2}$ y su resistencia virtual a cortante $\mathbf{f}_{\mathrm{cv}}=0,5 \mathrm{x} \quad 116=5,4 \mathrm{kp} / \mathrm{cm}^{2}$, de donde, la segura, resulta ser la de $\mathbf{f}_{\mathrm{v}}=5,4 / 1,6=3,4 \mathrm{kp} / \mathrm{cm}^{2}$. Sin estribos podría llegarse a los $6,8 \mathrm{kp} / \mathrm{cm}^{2}$. La tensión media resulta $16.200 / 1.259=\mathbf{1 2 , 9} \mathrm{kp} / \mathrm{cm}^{2}$, que supone $3,8 \mathbf{f}_{v}$, superior, incluso, a lo permitido con la máxima densidad de estribos. Desgraciadamente éstos, con la formulación actual, son poco eficaces, por lo que aquí se considerará que no existen.

Canto del forjado y lado del soporte, por este orden, son las variables que controlan básicamente el problema. El canto interviene favorablemente dos veces: una, en el perímetro crítico y otra en el canto de dicho perímetro, aunque su ventaja se ve algo reducida por el aumento de peso propio que suele significar. Incrementando el canto hasta $20 \mathrm{~cm}$, la carga total puede aumentar a $0,68 \mathrm{t} / \mathrm{m}^{2}$ y el cortante a 17 t. A cambio el canto mecánico a punzonado, d, sube a $14,2 \mathrm{~cm}$ y el lado crítico, b, a $27,8 \mathrm{~cm}$, lo que supone una sección crítica de $1.579 \mathrm{~cm}^{2}$ con lo que la tensión baja a $17.000 / 1.579=\mathbf{1 0 , 8} \mathrm{kp} / \mathrm{cm}^{2}$. El lado del soporte interviene sólo una vez en el proceso anterior, en el lado crítico. Si el lado del fuste, a, aumenta a $100 \mathrm{~mm}$, el lado crítico sube a $29,8 \mathrm{~cm}$, la sección a $1.681 \mathrm{~cm}^{2}$ y la tensión desciende, si bien no tan aparatosamente, a $17.000 / 1.681=10,1 \mathrm{kp} / \mathrm{cm}^{2}$. Sigue sin valer.

En ocasiones no se puede actuar sobre el canto del forjado, por la repercusión que tendría en el coste de la planta, y tampoco en el ancho del fuste, ya que, para la compresión existente, no queda margen para compensarlo con el espesor del tubo. Los casos más delicados son los de la última planta, en los que la compresión es pequeña, demandando un soporte de poca sección. En esos casos, la única variable sobre la que queda por actuar es el tamaño del perfil del collarín. Su efecto es ambiguo. Por un lado aumenta el lado del perímetro crítico, ya que lo aleja del soporte, pero por otro disminuye el canto crítico. En el ejemplo, disponiendo un perfil PNL50, el valor c aumenta a $3,5 \mathrm{~cm}$, lo que merma el canto mecánico a $13,5 \mathrm{~cm}$ pero consigue aumentar el lado crítico a $30,5 \mathrm{~cm}$. No obstante la sección crítica resultante, aunque poco, disminuye a $1.647 \mathrm{~cm}^{2}$, lo que parece indicar que es un cambio no recomendable ${ }^{4}$ (Figura 6).

Sucede que hasta ahora sólo se ha considerado la tensión de tracción o tangencial en el cono de punzonado. Falta considerar la de compresión en el plano de acometida, es decir, la de la biela en la cara superior del collarín. Y para ello sí resulta ventajoso aumentar su tamaño. La resultante de la biela que acomete al soporte tiene, como componente vertical, el cortante que entra en esa cara; entre las cuatro el total es $\mathbf{V}$, el incremento de carga del soporte a causa de

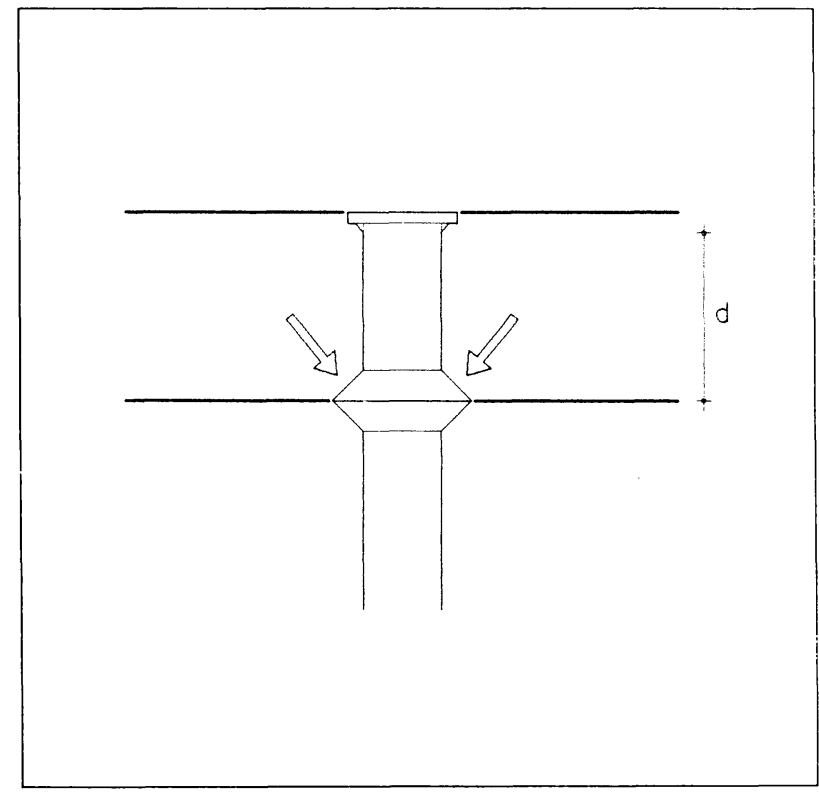

Figura 6.- Collarin descolgado.

ese forjado. Trazando desde la arista del collarín un plano perpendicular a la biela, lo que queda entre ese plano, el soporte y el propio collarín es un volumen confinado y tricomprimido, que no impone límites prácticos a su resistencia, aunque la propia biela, en su encuentro con el plano trazado, está parcialmente tricomprimida, a falta de convención normalizada en el tema ${ }^{5}$, parece prudente limitar su capacidad a la de la resistencia a compresión, $\mathbf{f}_{\mathrm{cd}}$. Si bien cuando la organización del forjado es unidireccional con vigas planas, cabe esperar que la carga acometa más por una dirección que por la otra, en primera aproximación, y tratándose de cálculos a pequeña distancia del fuste, con soportes interiores se puede suponer que lo hace por igual en todas las direcciones. En ese caso la tensión de compresión de la biela junto al collarin, tomando las componentes correlativas de dividendo y divisor, es, en alzado, $\mathbf{V} / \mathbf{c}, \mathrm{y}$, por tanto, la condición limitativa por aplastado resulta $V_{d} / 4(a+c) c<f_{c d}$.

En el ejemplo,el incremento de compresión del soporte era $17 \mathrm{t}$, resultando la sección de aplastado, por tanto, $4 \times(10+3,5) \times 3,5=189 \mathrm{~cm}^{2}$, lo cual significa tensiones del orden de $17.000 / 189=90 \mathrm{kp} / \mathrm{cm}^{2}$, superiores al valor deducido antes, de $73 \mathrm{kp} / \mathrm{cm}^{2}$, correspondiente a H- 175 . Aumentar el perfil de collarín para aliviar la compresión de la biela, es suicida desde el punto de vista del punzonado y al revés. Puesto que un solo parámetro, el ancho del ángulo, debe controlar dos condiciones, en algún caso, como éste, puede no haber solución. Para resolver el ejemplo es preciso retroceder, de nuevo, a las variables más relevantes: el canto del forjado y el del soporte. Con un forjado de $24 \mathrm{~cm}$, el peso de la planta asciende a $0,7 \mathrm{t} / \mathrm{m}^{2}$, lo cual significa que $\mathbf{V}=0,7 \times 25=17,5 \mathrm{t}$. Con un fuste de $\mathbf{a}=12 \mathrm{~cm}$, el canto eficaz a punzonado es $\mathbf{d}=$ $\mathbf{h}-\mathbf{c}-\mathbf{r}=17,5 \mathrm{~cm}$; el lado crítico es $\mathbf{b}=\mathbf{a}+\mathbf{2} \mathbf{c}+\mathbf{d}=36,5 \mathrm{~cm}$, 
lo que conduce a una sección crítica $\mathbf{S}=4 \mathbf{b ~ d}=2.555 \mathrm{~cm}^{2}$ y a una tensión tangencial ${ }^{6} \mathrm{~d}$ e $17.500 / 2.555=6,8 \mathrm{kp} / \mathrm{cm}^{2}$, que entra en lo soportable. Para comprobación de aplastado, la sección es $4 \mathbf{c}(\mathbf{a}+\mathbf{c})=217 \mathrm{~cm}^{2}$ lo que conduce a una tensión en las bielas de acometida de 17.500/217 = $80,6 \mathrm{kp} / \mathrm{cm}^{2}$, que todavía es algo fuerte para $\mathrm{H}-175$.

Aumentar la resistencia del hormigón mejora la capacidad de las bielas en la misma proporción, pero apenas cambia la resistencia a tensión tangencial. El paso a un hormigón $\mathrm{H}$-200 puede resolver el caso si el problema era el aplastado de la biela. Aunque en general no es recomendable aumentar la clase de hormigón de toda la estructura sólo por un problema puntual, en la actualidad el tipo ya es H-200 y, en un futuro próximo, parece que se pedirá $\mathrm{H}-250$; todo apunta a que, aunque el control no consiga demostrar que el hormigón es más que H-200, el ejemplo parece solucionado.

Los anteriores cálculos deben tomarse sólo como orientación. Es posible que en la próxima versión de $\mathrm{EH}$ (conocida entre los iniciados como EHE) cambie el valor de la resistencia a tensión tangencial, el valor de comparación para compresión en bielas confinadas $\mathrm{y}$, lo que será aún más trascendente, la distancia a la que se traza el perímetro crítico a punzonado, que, de medio canto, puede pasar a $\operatorname{dos}^{7}$ cantos. Aunque en ese último caso la tensión de comparación baje de $2 \mathbf{f}_{\mathrm{cv}}$ a $1 \mathbf{f}_{\mathrm{cv}}$, puede comprobarse que la combinación de ambos factores aumentará la capacidad resistente del problema planteado ${ }^{8}$.

En la tabla 1 aparece un resumen de los valores obtenidos, y algunos más. Con fustes de $80 \mathrm{~mm}$ de lado y collarines de PNL40 se puede soportar el equivalente a $12 \mathrm{~m}^{2}$ de vivienda; para llegar a $16 \mathrm{~m}^{2}$ es preciso contar, como mínimo, con $100 \mathrm{~mm}$ de fuste y collarines de PNL 45; si, como en el ejemplo, se quiere llegar a $25 \mathrm{~m}^{2}$ el soporte deberá tener un mínimo de $120 \mathrm{~mm}$ de lado y el collarín deberá ser de PNL50. Con cargas más elevadas o esbelteces de forjado menos usuales, como las de losa maciza, los valores de referencia pueden ser algo distintos.

Falta aún una comprobación adicional o, si se prefiere, un cálculo más: el de la soldadura entre collarín y fuste. En la práctica no supone limitación alguna. Por la posición del collarín, la compresión de la biela entra oblicuamente al fuste, lo que significa que el plano de soldadura está simultáneamente comprimido, lo que mejora su capacidad a cortadura. Si la biela es a $45^{\circ}$, ambos cordones se reparten por igual la carga; aun para variaciones fuertes de ese ángulo, el reparto difiere poco de esa proporción. Los perfiles PNL tienen, por lo general, un lado igual a 10 veces su espesor, t. En las comisuras entre ángulo y fuste puede albergarse con comodidad un cordón con un ancho de garganta $\mathbf{t} / \mathbf{2}$. Debido a la limitación por aplastado de las bielas, por unidad de longitud de ángulo, la compresión sobre su ala no puede exceder de $\mathbf{f}_{\mathrm{c}} 10 \mathrm{t}$, de donde la tensión en cada uno de los dos cordones no supera $10 \mathbf{f}_{q}$. Ese valor se encuentra en la banda de $800 \mathrm{kp} / \mathrm{cm}^{2}$, mientras que, en el peor de los casos, la resistencia segura de la soldadura en A42 es, al menos, $1.200 \mathrm{kp} / \mathrm{cm}^{2}$. La soldadura no es problema ${ }^{9}$.

Si la estructura está sometida a acciones horizontales o la situación del soporte no es simétrica en luces, el problema debe reformularse para aceptar momentos en los soportes. En ese caso, mejor que considerar tensiones tangenciales en distribución variable, es transformar la solicitación del soporte en una compresión excéntrica, y proceder con el modelo anterior en torno a la resultante de las compresiones, que no será el centro del soporte, pero no podrá alejarse mucho $^{10}$ de él.

TABLA 1.- CAPACIDAD SEGURA DE COLLRINES EN SOPORTES INTERIORES CON CARGA CENTRADA, HORMIGÓN H-175

\begin{tabular}{|c|c|c|c|c|c|c|c|c|}
\hline $\begin{array}{l}\text { Lado del } \\
\text { soporte } \\
(\mathrm{mm})\end{array}$ & $\begin{array}{l}\text { Perfil } \\
\text { de } \\
\text { ángulo }\end{array}$ & $\begin{array}{l}\text { Vuelo } \\
\text { c } \\
(\mathrm{cm})\end{array}$ & $\begin{array}{l}\text { Canto del } \\
\text { forjado } \\
\text { h } \\
(\mathrm{cm})\end{array}$ & $\begin{array}{l}\text { Canto } \\
\text { mecánico } \\
\mathbf{d = h}-\mathbf{r}-\mathbf{c} \\
(\mathrm{cm}) \mathrm{r}=3\end{array}$ & $\begin{array}{l}\text { Lado } \\
\text { crítico } \\
b=\mathbf{a}+2 c+d \\
(\mathrm{~cm})\end{array}$ & $\begin{array}{l}\text { Sección } \\
\text { crítica } \\
S=4 b d \\
\left(\mathrm{~cm}^{2}\right)\end{array}$ & $\begin{array}{l}\text { Capacidad } \\
\text { punzonado } \\
V=2 \mathbf{C}_{\mathbf{L}} \mathbf{S} \\
\text { (t) }\end{array}$ & $\begin{array}{l}\text { resistente por } \\
\text { aplastado } \\
\begin{array}{l}V=f_{c} 4 c(a+c) \\
(l)\end{array}\end{array}$ \\
\hline $\begin{array}{c}70 \\
80 \\
80 \\
80 \\
100 \\
100 \\
100 \\
100 \\
100 \\
120 \\
140\end{array}$ & $\begin{array}{l}\text { PNL35 } \\
\text { PNL35 } \\
\text { PNL40 } \\
\text { PNL40 } \\
\text { PNL40 } \\
\text { PNL45 } \\
\text { PNL45 } \\
\text { PNL50 } \\
\text { PNL50 } \\
\text { PNL50 } \\
\text { PNL50 }\end{array}$ & $\begin{array}{l}2,5 \\
2,5 \\
2,8 \\
2,8 \\
2,8 \\
3,1 \\
3,1 \\
3,5 \\
3,5 \\
3,5 \\
3,5\end{array}$ & $\begin{array}{l}16 \\
18 \\
18 \\
20 \\
20 \\
20 \\
22 \\
22 \\
24 \\
24 \\
24\end{array}$ & $\begin{array}{l}10,5 \\
12,5 \\
12,2 \\
14,2 \\
14,2 \\
13,9 \\
15,9 \\
15,5 \\
17,5 \\
17,5 \\
17,5\end{array}$ & $\begin{array}{l}22,5 \\
25,5 \\
25,8 \\
27,8 \\
29,8 \\
30,1 \\
32,1 \\
32,5 \\
34,5 \\
36,5 \\
33,5\end{array}$ & $\begin{array}{r}945 \\
1.275 \\
1.259 \\
1.579 \\
1.681 \\
1.673 \\
2.041 \\
2.015 \\
2.415 \\
2.555 \\
2.695\end{array}$ & $\begin{array}{r}6,4 \\
8,7 \\
8,6 \\
10,6 \\
11,4 \\
11,4 \\
13,9 \\
13,7 \\
16,4 \\
17,4 \\
18,3\end{array}$ & $\begin{array}{c}6,9 \\
7,7 \\
8,8 \\
8,8 \\
10,4 \\
11,8 \\
11,8 \\
13,8 \\
13,8 \\
15,8 \\
17,9\end{array}$ \\
\hline
\end{tabular}


Una situación límite de asimetría es la del soporte de borde. Dada la desproporción de rigideces y, sobre todo, la disposición habitual y casi obligada de triangulaciones globales en la estructura, se puede considerar el forjado pasante sobre los soportes, es decir, apoyo simple en ese punto, exento de momento. Como la solución habitual y recomendable es que la tabica del forjado sobresalga unos centímetros de la cara exterior del soporte, en esos puntos se puede mantener la solución constructiva del collarín ETSAM, aunque varíe su capacidad.

Por punzonado, observando la pérdida de tronco de pirámide de la superficie crítica (Figura 7), es inmediato que la capacidad de un collarín de borde se reduce algo más de la mitad de la del caso interior. Por aplastado de bielas puede quedar una capacidad residual de hasta tres cuartas partes (el ángulo del lado del borde no puede recibir carga).

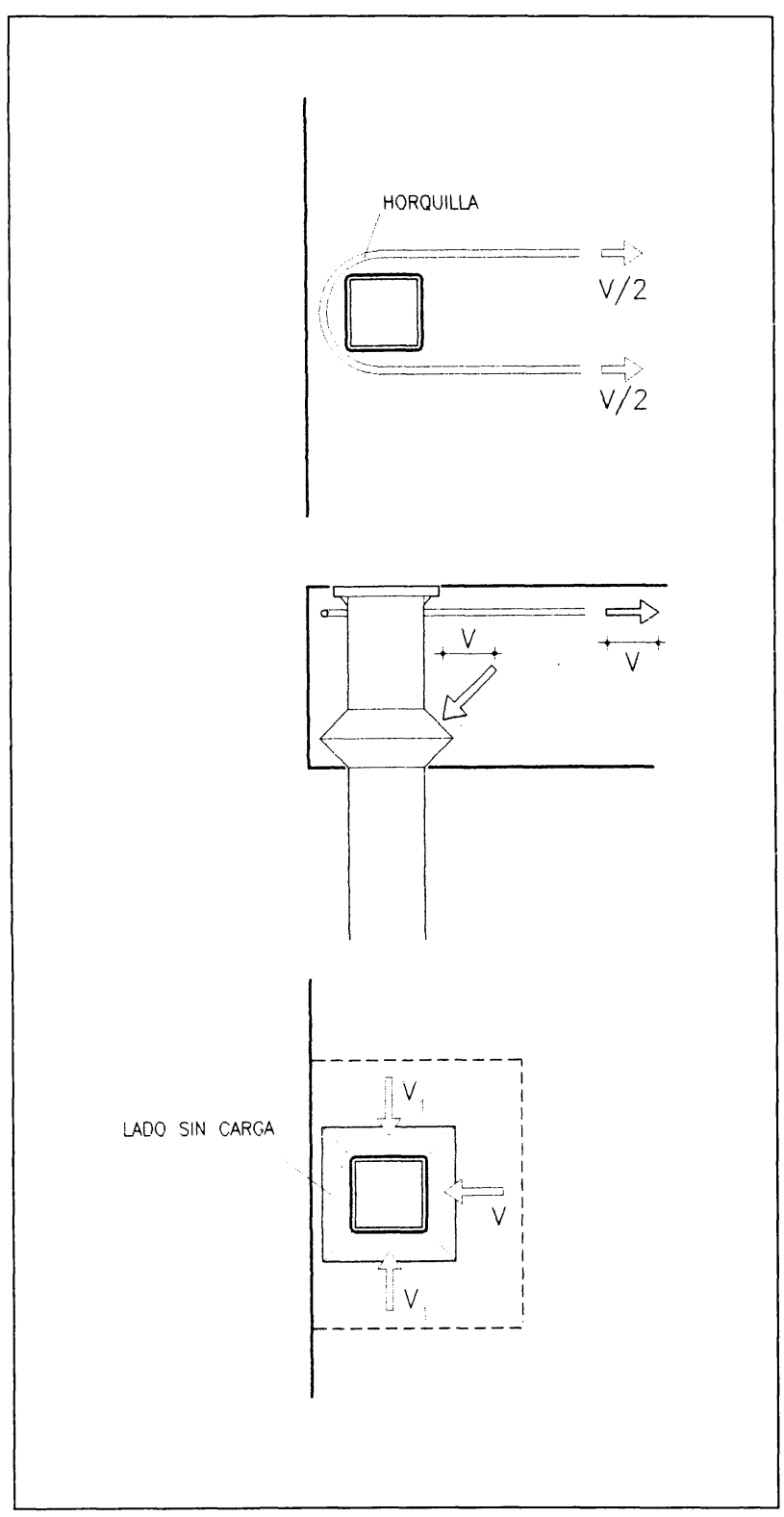

Figura 7.- Soporte de borde.
Ahora tendría más sentido discutir acerca de la organización resistente del forjado. Si se trata de forjado unidireccional con vigas planas, y la viga es paralela al borde, el collarín apenas pierde capacidad por aplastado de bielas. Si es perpendicular al borde podría verse reducida a la mitad. Como de todas maneras la carga que debe entrar en el soporte también es del orden de la mitad de la del caso anterior, la situación de este collarín no es peor que la del estudiado, si no fuera porque el soporte tendrá tendencia también a tener menos sección.

El aspecto peculiar de este caso de borde es que la situación de tensión está desequilibrada. La biela perpendicular al borde no encuentra oposición, y todo lo estudiado fracasaría, a menos que se disponga, en la parte superior del forjado, una tracción de valor igual a la componente horizontal de la compresión de la biela. Para bielas a $45^{\circ}$ sería una tracción igual al cortante que entra por esa cara. Dicha tracción debe anclarse en el soporte. La solución más simple es disponerla con dos redondos, anclándolos uno contra otro, por detrás del soporte, en horquilla. Cabe también doblarlos en escuadra, anclándolos a un redondo o perfil transversal situado en la parte superior, paralelo al borde $\mathrm{o}$, incluso, directamente soldados a las caras laterales del fuste, pero ésas son soluciones más complejas que la de la horquilla. Para soportes dispuestos cada $5 \times 5 \mathrm{~m}$ en vivienda, la carga total que entra en un soporte ${ }^{11}$ de borde sería del orden de $0,7 \times 5 \times 5 / 2=8,7 \mathrm{t}$, de la que, por la cara interior, entran $8,7 / 2=4,3 \mathrm{t}$. Una tracción como ésa en acero AEH500 queda resuelta con $4,3 / 2,8=1,5 \mathrm{~cm}^{2}$, es decir, una horquilla de 2 ramas de $\phi 10$. Por lo general no se baja de $\phi 12$ y es muy raro el caso que no pueda resolverse con una horquilla de $\phi 16$.

El problema del soporte de esquina (Figura 8) es similar. Lo peor vendrá de la mano de que el soporte suele ser de mucha menos sección, pero por lo que respecta al collarin, puede disponerse el mismo, conservando la cuarta parte de la capacidad a punzonado, y la mitad de la de aplastado de bielas, que suele ser suficiente para una entrada de carga que es del orden de la cuarta parte de la del soporte interior con las mismas luces. Ahora son dos los ángulos del collarín que no reciben carga y la asimetría es más acusada. Con losa maciza lo aconsejable sería disponer una horquilla en la bisectriz del ángulo exterior, complementada con alguna armadura secundaria perpendicular; con forjados unidireccionales y vigas planas, podrá ser suficiente con una horquilla en la dirección de la viga.

Es posible que, en un futuro próximo, cambien los detalles de las comprobaciones de punzonado y demás aplicables al caso, pero en lo fundamental, y dado que los números se hacen como ayuda para tomar las decisiones, cabe esperar que el collarín ETSAM siga siendo una buena, sencilla y barata solución al problema de cómo engarzar soportes metálicos a forjados de hormigón armado para obras de arquitectura. 


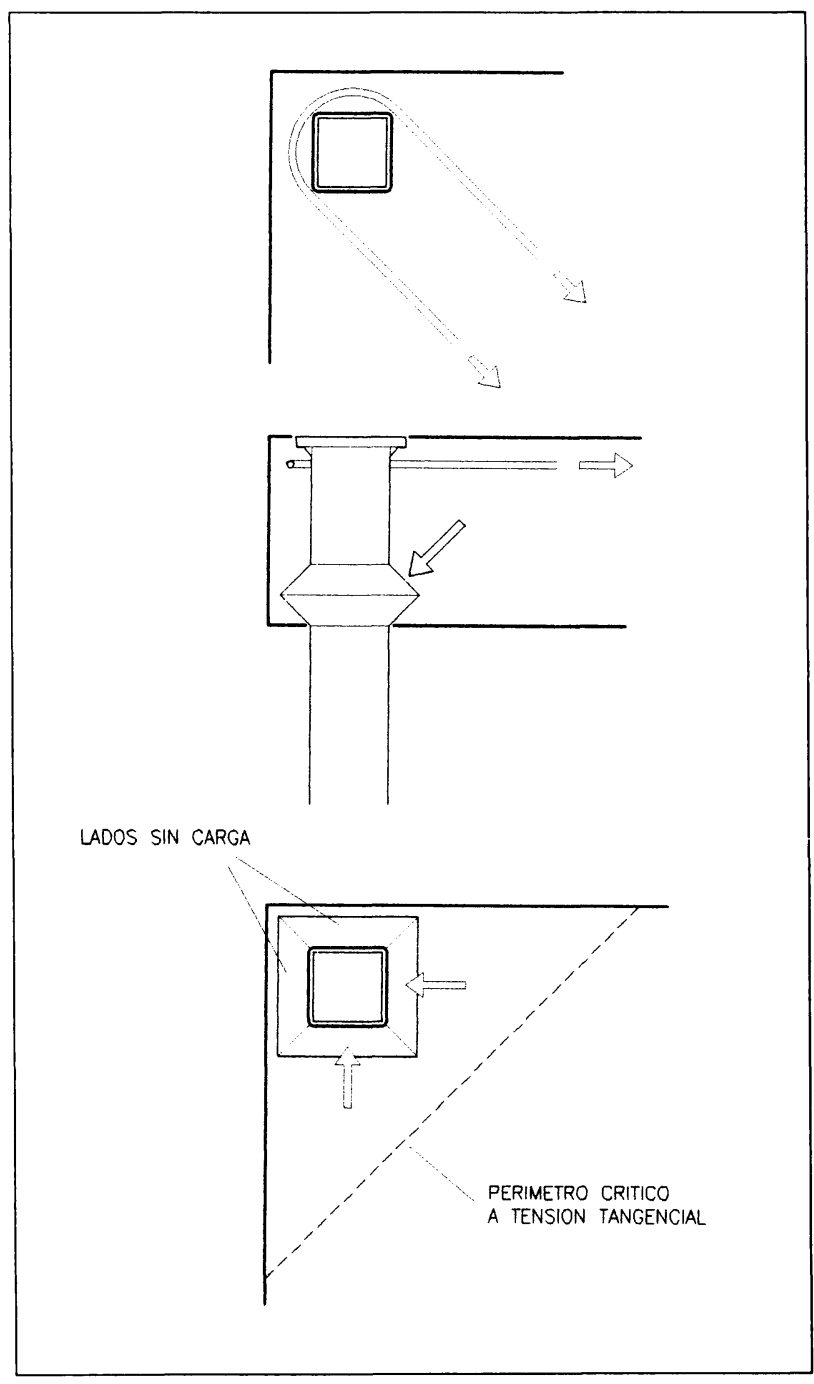

Figura 8.- Soporte de esquina.

\section{NOTAS}

1 El autor de este artículo, en 1982, aprovechando una obra en la que había que utilizar varios miles de estos collarines, pudo realizar un ensayo a escala real, poniendo a prueba cuatro soportes: interior, lateral, frontal y esquina, superando con éxito 2,4 veces la carga de servicio sin síntomas de fracaso. Aunque todavía en algunos círculos esta solución se califica de "poco usual", esperemos que, tras esta publicación, deje de serlo.

${ }^{2}$ En rigor, es la línea de menos perímetro, a no más de medio canto mecánico del área cargada.
${ }^{3} \mathrm{Al}$ menos al autor de este artículo le parece evidente. No obstante ha visto recientemente informes, en los que se comprobaba este tipo de soluciones, que introducían, en un programa de comprobación de punzonado, el lado a del soporte metálico, como lado del área cargada, sin ninguna justificación y sin que luego se pudiese saber qué se hacía con los resultados.

${ }^{4}$ Una solución alternativa es la de disponer la arista del collarín en el plano inferior del forjado, lo que iguala el canto eficaz al total. Aunque a veces no es compatible con el resto de la construcción y el encofrado es más delicado, su aspecto es impecable.

${ }^{5}$ Es muy posible que la próxima versión de EH incluya una lista exhaustiva de capacidad a compresión de bielas en múltiples situaciones, a alguna de las cuales pueda asimilarse ésta.

${ }^{6}$ En rigor, a la carga total, de $17.500 \mathrm{kp}$, se le puede restar la que existe dentro del perímetro crítico, que resulta, en este caso, 700 ×0,36×0,36 =91 $\mathrm{kp}$; el error, del lado de la seguridad, no merece la pena.

${ }^{7}$ Que el perímetro crítico a tensión tangencial se encuentre a dos cantos, significa que el de tracción equivalente llega a cuatro cantos. En arquitectura cuatro cantos invadirá, con frecuencia, la zona de bovedillas o viguetas, perdiéndose fiabilidad, ya que el usuario no estará advertido. Por otro lado calcular el cortante a un canto y el punzonado a dos, dará lugar a efectos inesperados en los casos de forjados unidireccionales con vigas planas.

${ }^{8}$ Con EH-91 el problema de punzonado afecta casi en exclusiva al caso de soportes de acero. Si se consolidan los cambios previstos en EHE, la tensión tangencial por punzonado es soportable aun con soportes tan pequeños como un punto si la carga es la de edificios usuales. Con soportes de hormigón, para que haya problemas de tensión tangencial por punzonado habrá que rebasar muy ampliamente las cargas máximas de las obras de arquitectura. En este tipo de construcciones, del problema sólo subsistirá la compresión de las bielas cuando el soporte es de acero, con expresiones que están pendientes de formular.

9 En algún caso se ha argumentado que otro problema podría ser el del fracaso a flexión de la propia pared del ala del ángulo. Aun considerando los bordes como apoyos simples, para las proporciones de los ejemplos, considerando flexión bidireccional, cabe esperar un momento máximo del orden de $q \mathbf{L}^{2} / 13$. Siendo $q=f_{c}$ y $\mathbf{L}=8 \mathbf{t}$ resulta un momento $5 f_{\mathrm{c}} \mathbf{t}^{2}$. El módulo resistente plástico de una chapa de espesor $\mathbf{t}$, es $\mathbf{t}^{2} / 4$ de donde la tensión máxima no superaría 20 f. Como f está en la banda de $73 \mathrm{kp} / \mathrm{cm}^{2}$ y la tensión segura del acero A42 es del orden de $1.800 \mathrm{kp} / \mathrm{cm}^{2}$, la condición tampoco es limitativa.

${ }^{10}$ Incluso considerando que toda la acción de viento se soporta por efecto de pórtico, a base de flexiones en los soportes, de las obras de arquitectura usuales la trayectoria de carga en el soporte rara vez se descentra más de $3 \mathrm{~cm}$.

11 Los soportes deben calcularse para la solicitación resultante de las bielas más la horquilla. Eso cobra especial importancia en los de la última planta, en el que el momento de la horquilla debe ser soportado por el fuste incluido en el grueso del forjado, que no puede ser eliminado y que debe poseer un retallo para evitar la expulsión de la horquilla. 